Advances in Radio Science (2004) 2: 79-82

(c) Copernicus GmbH 2004

\title{
HPEM protection on HF transmission lines
}

\author{
R. Krzikalla and J. L. ter Haseborg
}

TU Hamburg-Harburg, Arbeitsbereich Messtechnik/EMC Harburger Schloss-Strasse 20, 21071 Hamburg, Germany

\begin{abstract}
Traditional protection concepts against electromagnetic interferences are combinations of nonlinear elements like spark gaps, varistors or suppressor diodes. Due to parasitic capacitances and inductivities and the general behavior of the elements, a trade off has to be made between the frequency bandwidth and the energy absorption capability of the protection circuit. For high frequency applications with signal frequencies above about $500 \mathrm{MHz}$ a sufficient protection can not be given without changing the transmitted signals itself. Additionally this kind of protection concept is designed and optimized against transient interferences with rise times down to some nanoseconds, for example the LEMP, the NEMP or the ESD. Current researches focus on transient interferences with rise times in the picosecond range and pulse widths of a few nanoseconds, the so called ultra wideband (UWB) pulses, combined with a significant voltage amplitude of some kilovolts. In this paper investigations on nonlinear protection elements and linear filters against line-coupled UWB pulses with high voltage amplitudes will be done with special focus on the protection of high frequency data-lines.
\end{abstract}

\section{Introduction}

Electronic systems became an essential part in the civil life but also in solving military problems. The rising requirements on communication bandwidths go along with the increase of the used signal frequencies. While electronic systems can be protected against electromagnetic fields with sufficient shielding methods, line coupled interferences on power or data lines can have effects inside the system. Many investigations have been done for the well known transient interferences like the LEMP, the NEMP or the ESD. Current works focus transients with rise times in the picosecond range and pulse widths of a few nanoseconds. Combined with voltage amplitudes of some kilovolts these so called ul-

Correspondence to: R. Krzikalla

(krzikalla@tu-harburg.de) tra wideband (UWB) pulses are a serious threat for modern electronic systems (Radasky et al., 2003; Camp et al., 2002).

While interferences like the LEMP, the NEMP or the ESD have already been standardized, the standardization of UWB pulses is still in progress. Therefore in this paper a worst case approximation on line coupled UWB pulses will be assumed. According to other descriptions of electromagnetic interferences (Standler, 1989), also UWB pulses will be described in time domain with a double-exponential function:

$u_{\mathrm{UWB}}(t)=V_{p} \cdot k \cdot\left[e^{-\alpha \cdot t}-e^{-\beta \cdot t}\right]$

$\alpha$ and $\beta$ set up the rise time and the pulse width and $k$ is a normalization factor, so that $V_{p}$ becomes the voltage maximum of the pulse. Values for the rise times of worst case line coupled ultra wideband pulses are below $200 \mathrm{ps}$ and the pulse widths are shorter than $5 \mathrm{~ns}$. Therefore UWB pulses are much faster and shorter than all other known electromagnetic interferences. Table 1 compares rise times and pulse widths of some selected natural and man-made electromagnetic interferences. With these values the corresponding frequency spectrum of ultra wideband pulses goes up to about $10 \mathrm{GHz}$.

\section{Measurement setup}

The general measurement setup to examine the behavior of protection elements by stressing with UWB pulses consists of an UWB pulse generator, the device under test and an oscilloscope. Due to the high frequency bandwidth of the ultra wideband pulse combined with voltage amplitudes in the kilovolt range, high demands on the measurement equipment have to be made. In Weber and ter Haseborg (2002), a measurement setup is presented and discussed, that provides a high frequency bandwidth with high voltage resistance. Figure 1 shows the used setup with the measurement probe "picoTEM".

At this time there are no UWB pulse generators available that maintain the time constants of the output signal for variable output voltages. To provide UWB pulses with voltage amplitudes below and above the breakdown voltage of 
Table 1. Pulse parameters for exemplary pulse forms (Standler, 1989).

\begin{tabular}{ccc}
\hline pulse type & rise time & pulse width \\
\hline LEMP & $10 \mu \mathrm{s}$ & $700 \mu \mathrm{s}$ \\
NEMP & $5 \mathrm{~ns}$ & $300 \mathrm{~ns}$ \\
ESD & $1 \mathrm{~ns}$ & $30 \mathrm{~ns}$ \\
UWB & $<100 \mathrm{ps}$ & $<5 \mathrm{~ns}$ \\
\hline
\end{tabular}

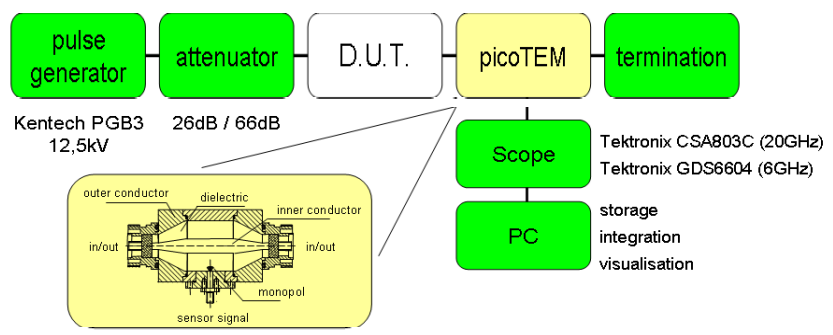

Fig. 1. Measurement setup to determine the pulse response of protection elements with high electric strength and high frequency bandwidth (Weber and ter Haseborg, 2002).

nonlinear protection elements, in this setup a $12.5-\mathrm{kV}-\mathrm{UWB}$ pulse generator has been used in combination with a $26 \mathrm{~dB}$ - and a $66 \mathrm{~dB}$ - attenuator. These test pulses, named as Ref26dB and Ref66dB, are shown in Fig. 2. The voltage amplitudes of the test pulses are $690 \mathrm{~V}$ and $6.9 \mathrm{~V}$, respectively. The rise time of both pulses is $130 \mathrm{ps}$ and the pulse width is about $2.2 \mathrm{~ns}$.

\section{Protection elements}

Protection elements have to suppress an occurring interference on the line without changing the wanted signal. Protection concepts against line coupled electromagnetic interferences normally built up as combinations of nonlinear elements like spark gaps, varistors or diodes (Bogeest, 1998). These protection circuits are designed and optimized against interferences with rise times down to some nanoseconds. Also a tradeoff has to be made between the energy absorption capability and the limiting bandwidth of the protection circuit (Bogeest, 1998). A broad frequency bandwidth leads to a quite low energy absorption capability and vice versa. Also the time response of most of the used protection elements is in the same range as the rise time of UWB pulses. Therefore the selected protection elements will be characterized in time domain by stressing with the presented UWB test pulses as well as in frequency domain to show the possibility of the usage in high frequency applications.

\subsection{Nonlinear elements}

As mentioned before traditional concepts against electromagnetic interferences consist of combinations of spark gaps, varistors and diodes. Due to the characteristics of spark
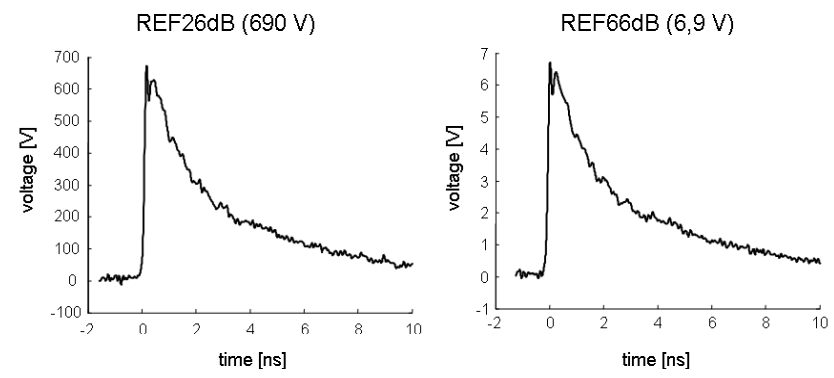

Fig. 2. Used UWB test pulses with amplitudes above and below the breakdown voltage of nonlinear elements.

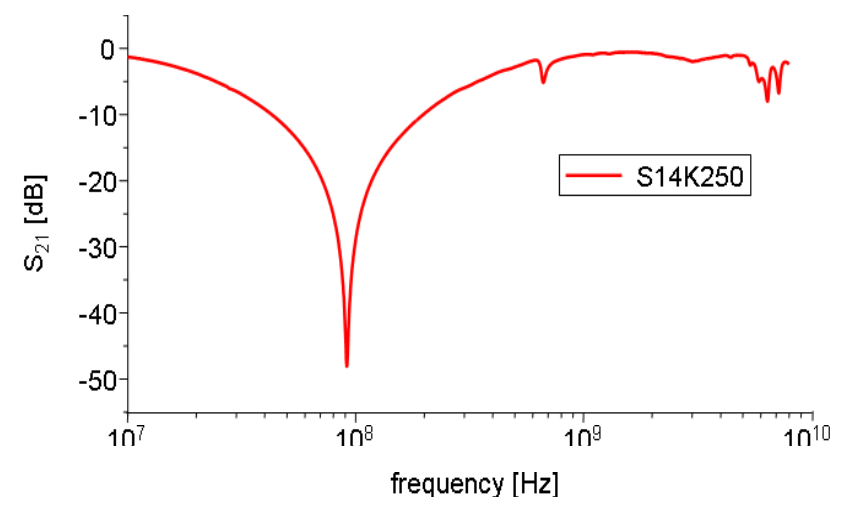

Fig. 3. Frequency response of a varistor S14K250 (manufacturer: EPCOS).

gaps where the breakdown voltage increases with the decreasing rise time of the interference significant effect will be assumed by stressing with UWB pulses. This could be confirmed in experiments, whereby spark gaps will not take part in this investigation. In the following selected nonlinear protection elements will be tested, that could be capable against UWB interferences due to their high energy absorption capability or their fast response time.

\subsubsection{Varistors}

Varistors are used in nonlinear protection circuits due to their high energy absorption capability. In combination with line inductivities varistors show lowpass behavior. Figure 3 shows the frequency response of a varistor of the type S14K250 (manufacturer: Epcos). Note that the attenuation at frequencies higher than $500 \mathrm{MHz}$ is nearly $0 \mathrm{~dB}$ again. The resulting time response of the selected varistor stressed by an UWB pulse is shown in Fig. 4. There is a significant reduction of the overall energy content of the interfering pulse due to the varistor, but the rising edge of the pulse has not been influenced. Furthermore Fig. 4 shows the time response with reduced voltage amplitude of the UWB pulse. It is remarkable that the results are very similar and hence the behavior of the varistor is not nonlinear in nature. Linear effects caused by the capacitance and feed line inductivities of the varistor can rather describe the resulting time responses. 

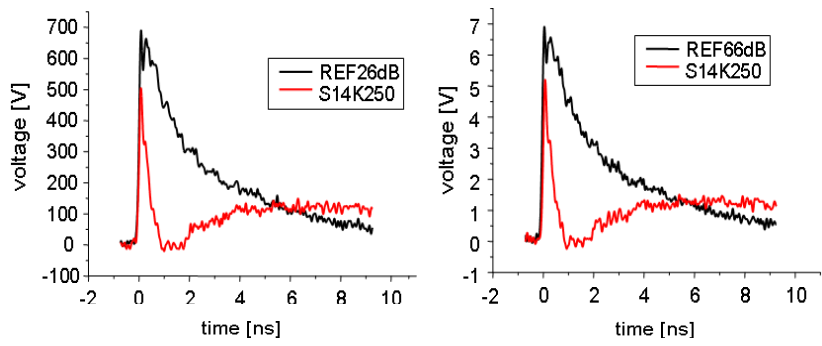

Fig. 4. Measured time response of a varistor (manufacturer: EPCOS) by stressing with the UWB pulses REF26dB and REF66dB

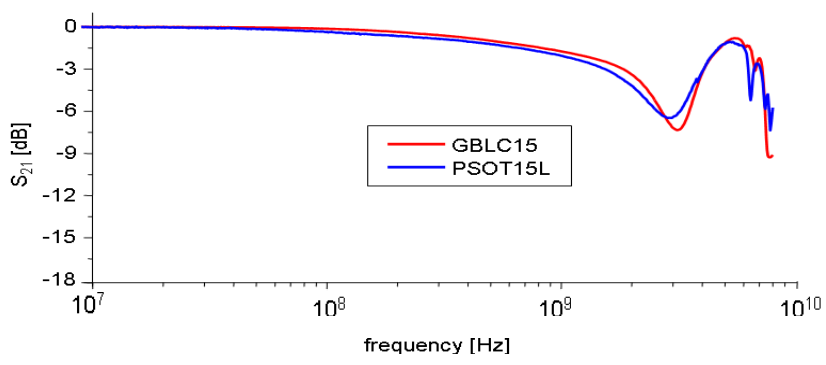

Fig. 5. Transfer function of the chosen SMD diodes PSOT15L and GBLC15C.

\subsubsection{Suppressor diodes}

Diodes are used as fine protection elements in nonlinear protection circuits. The presented suppressor diodes have been selected due to their very small parasitic capacitances and their very fast response times. The frequency responses show a very broad bandwidth (see Fig. 5), so that the usage in high frequency applications up to $2 \mathrm{GHz}$ is possible. The breakdown voltage of both of the diodes is $15 \mathrm{~V}$. Figure 6 shows the time response of the diodes by stressing with UWB pulses above and below the breakdown voltage. Again a reduction of the energy of the UWB pulse with a voltage amplitude can be noticed. Also the rising edge of the test pulse has not been influenced significantly. But in contrast to the tested varistor, the diodes do not show any significant changes of the pulse shape of the interference. Therefore the diodes behave in contrast to the varistor in a nonlinear way.

\subsection{Linear elements}

According to the linear behavior of many nonlinear elements by stressing with UWB pulses directly linear filters are developed to suppress such extremely fast interferences. Most applications are only using a specific frequency band. The ideal linear protection method for band limited applications is the use of bandpass filters. The energy of an interference can be reduced to a minimum by using bandpasses with a very high filter order without influencing the wanted signal. Typical high frequency applications are for example the mobile telephony in the GSM900- and GSM1800-band or Bluetooth and wireless LAN in the ISM-band (Industrial- Scientific-
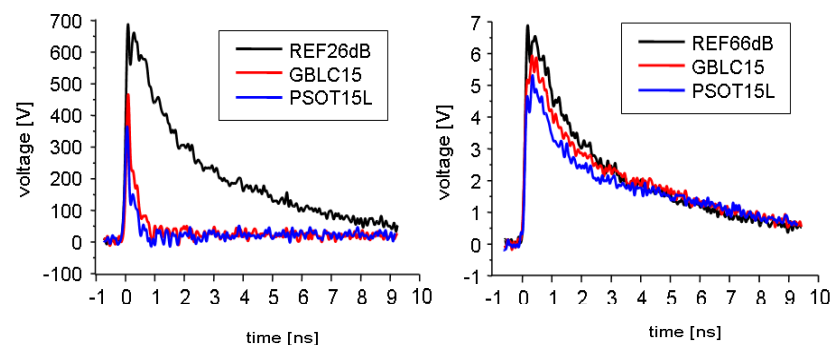

Fig. 6. Measured time response of the diodes PSOT15L and GBLC15C with UWB pulses of different voltage amplitudes.

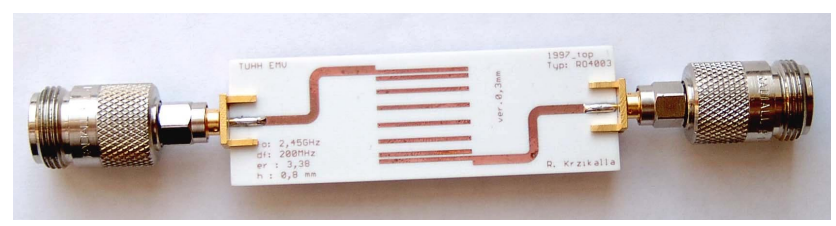

Fig. 7. Realisation of a 2.4-GHz-interdigital bandpass filter for the ISM band on a substrate RO4003 with the characteristics: $\varepsilon_{r}=3.38$, $\mathrm{h}=0.7 \mathrm{~mm}, \mathrm{t}=35 \mu \mathrm{m}, \tan \delta=0.0021$.

Medical) at $2.4 \mathrm{GHz}$. Table 2 gives a short overview of the rest energies of a $12.5 \mathrm{kV}$ UWB pulse for some selected high frequency applications. Furthermore the bandpasses have to be high voltage resistant und should be small and cost effective. Therefore microstrip filters have been designed. For a practical application an interdigital bandpass filter for the ISM-band (see Fig. 7) has been designed with very steep edges, a very high attenuation in the stopband and low losses in the passband. The measured transfer function and the time response of the interdigital bandpass filter by stressing with the UWB test pulse Ref26dB are shown in Fig. 8. The characteristics of the developed filter are summarized in Table 3. Due to the high filter order the edges of the filter are very steep, so that a high reduction of the pulse energy can be assumed. The UWB test pulse with a voltage amplutide of $690 \mathrm{~V}$ has been reduced to a damped sinusoid signal with a voltage amplitude of about $8 \mathrm{~V}$.

\section{Conclusion}

For the protection of high frequency applications against electromagnetic interferences nonlinear and linear elements have been tested. Especially diodes with a very small parasitic capacitance and a very fast response behavior are capable for high frequency applications against UWB pulses. While the energy of an UWB pulse could be reduced, the rising edge and the maximum voltage have not been influenced by the tested nonlinear elements significantly. Due to the linear behavior of nonlinearities with comparable high parasitic capacitances directly voltage resistant bandpass filters with high filter order have been developed to reduce the energy of an interference to a minimum without influence of the wanted signal on the line. 

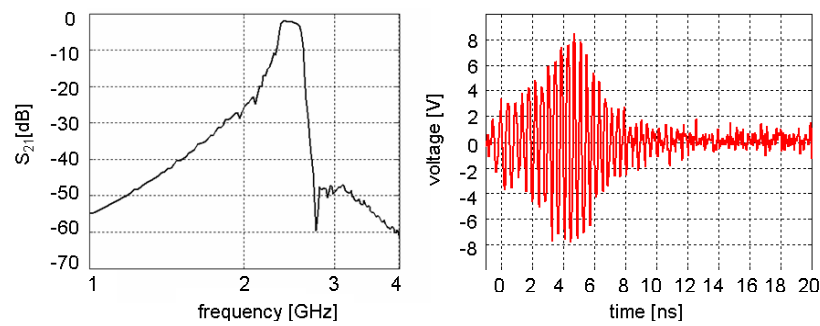

Fig. 8. Frequency response and filtered UWB pulse (Ref26dB) of the developed interdigital microstrip bandpass for the ISM band.

Table 2. Resulting energy of an UWB pulse (rise time $130 \mathrm{ps}$, pulse width $2.2 \mathrm{~ns}, V_{p}=12.5 \mathrm{kV}$ ) at $50 \Omega$ for different applications and different filter orders.

\begin{tabular}{ccc}
\hline $\begin{array}{c}\text { application } \\
\left(f_{0} / \Delta_{f}\right)\end{array}$ & $\begin{array}{c}\text { filter order } \\
(\text { Chebyshev })\end{array}$ & $\begin{array}{c}\text { rest energy } \\
50 \Omega\end{array}$ \\
\hline UWB pulse $12.5 \mathrm{kV}$ (unfiltered) & - & $4.3673 \mu \mathrm{J}$ \\
GSM900 (960 MHz/40 MHz) & 1 & $23.277 \mu \mathrm{J}$ \\
& 3 & $5.7651 \mu \mathrm{J}$ \\
GSM1800 (1.82 GHz/40 MHz) & 5 & $4.1463 \mu \mathrm{J}$ \\
& 1 & $2.6483 \mu \mathrm{J}$ \\
& 3 & $0.4751 \mu \mathrm{J}$ \\
ISM (2.45 GHz/100 MHz) & 5 & $0.2568 \mu \mathrm{J}$ \\
& 1 & $5.9125 \mu \mathrm{J}$ \\
& 3 & $0.9315 \mu \mathrm{J}$ \\
& 5 & $0.6625 \mu \mathrm{J}$ \\
\hline
\end{tabular}

Table 3. Characteristics of the developed interdigital bandpass filter for the ISM band.

\begin{tabular}{lc}
\hline center frequency & $f_{0}=2.45 \mathrm{GHz}$ \\
bandwidth & $\Delta_{f}=200 \mathrm{MHz}$ \\
filter order & $\mathrm{n}=6$ \\
max. ripple in the passband & $\alpha_{1}=0.1 \mathrm{~dB}$ \\
characteristic impedance & $R_{0}=50 \Omega$ \\
substrate & $\mathrm{RO} 4003$ \\
specific dielectric constant & $\epsilon_{r}=3.38$ \\
dissipation factor & $\tan (\delta)=0.0021$ \\
\hline
\end{tabular}

Acknowledgements. The authors would like to thank the BWB (Germany) and the WIS (Scientific Research Institute for Protection Technologies, Munster, Germany) for financial funding and support of this work.

\section{References}

Radasky, W. A., Messier, M., and Wik, M.: Intentional Electromagnetic Interference (EMI) - Test data and implications, International Zürich Symposium on EMC 2003, Zürich, Februar 2003.

Camp, M., Garbe, H., and Nitsch, D.: Influence of the technology on the destruction effects of semiconductors by impact of EMP and UWB pulses, IEEE Symposium on EMC, Minneapolis, 2002.

Standler, R. B.: Protection of electronic circuits from overvoltages, Wiley Interscience, New York, 1989.

Weber, T., and ter Haseborg, J. L.: A new broad-band probe for the measurement of ultra-fast transients, EMC Europe 2002, Sorrento, Italy, 2002.

Borgeest, K.: Optimierung und Simulation des transienten Ansprech- und Übertragungsverhaltens nichtlinearer Schutzschaltungen für HF-Systeme, Ph.D. thesis, Shaker Verlag, Aachen, 1998. 